\title{
Development and Characterization of Vitamin A-Loaded Solid Lipid Nanoparticles for Topical Application
}

\author{
Mauricio Argimón, ${ }^{a}$ Mariano Romero, ${ }^{a, b}$ Pablo Miranda, ${ }^{a}$ Álvaro W. Mombrú, ${ }^{a, b}$ \\ Iris Miraballes, ${ }^{c}$ Patricia Zimet ${ }^{a}$ and Helena Pardo ${ }^{*, a, b}$
}

\author{
${ }^{a}$ Centro NanoMat, Instituto Polo Tecnológico de Pando, Facultad de Química, UdelaR, \\ Camino Aparicio Saravia s/n, 91000 Pando, Canelones, Uruguay \\ ${ }^{b}$ Cátedra de Física, Facultad de Química, DETEMA, UdelaR, General Flores 2124, \\ 11800 Montevideo, Uruguay
}

\author{
'Laboratorio de Biotecnología, Instituto Polo Tecnológico de Pando, Facultad de Química, UdelaR, \\ Camino Aparicio Saravia s/n, 91000 Pando, Canelones, Uruguay
}

\begin{abstract}
Vitamin A and its esters are commonly found in topical applications because of their advantageous properties, however, have the drawback that are highly sensitive to ultraviolet radiation. The aim of this work was to develop and characterize a novel formulation of solid lipid nanoparticles suitable for topical applications in order to protect vitamin A from degradation. Vitamin A-loaded nanoparticles were successfully prepared by hot homogenization employing Gelucire $44 / 14^{\circledR}$ and cetyl alcohol as carrier materials, showing an entrapment efficiency of more than $90 \%$. Particle size, measured by dynamic light scattering, was ca. $40 \mathrm{~nm}$, while transmission electron microscopy images showed that dried nanoparticles were spherical with an average size of about 30-50 nm. Small angle X-ray scattering was used to study their aspect ratio and their physicochemical properties were evaluated by differential scanning calorimetry, infrared spectroscopy and X-ray powder diffraction, additionally, stability of vitamin A was studied by UV-Vis spectroscopy.
\end{abstract}

Keywords: solid lipid nanoparticles, vitamin A, topical delivery

\section{Introduction}

Vitamin A palmitate is a fat-soluble vitamin that is involved in the formation and maintenance of healthy skin, hair and mucous membrane. It helps to increase skin elasticity, reduce roughness and prevent peroxidation of skin lipids by exfoliating the top layer of the skin, which accelerates cell renewal, making the skin look fresher, smoother and younger. Also, vitamin A acts as an antioxidant, preventing tissue atrophy and collagen loss during aging. ${ }^{1}$ Because of its antioxidant and moisturizing properties, vitamin A is commonly found in creams or gels for topical use. However, it is highly sensitive to ultraviolet radiation and oxygen, which can rapidly promote its degradation leading to the decrease of the content of vitamin $\mathrm{A}$ in the epidermis. ${ }^{2}$ Therefore, an adequate drug delivery system is required to preserve the activity of this

*e-mail: hpardo@fq.edu.uy lipophilic compound. Solid lipid nanoparticles (SLNs) were developed at the beginning of the decade of 1990 as an alternative to other drug delivery systems such as polymeric particles, lipid emulsions and liposomes. In fact, this cutting-edge technology has attracted wide attention as colloidal drug carrier of lipophilic compounds for topical use, since it combines the advantages of other delivery systems. ${ }^{3}$ SLNs are a special type of nanoemulsion with sizes ranging from 50 to $1000 \mathrm{~nm}$, with a solid lipid matrix instead of a liquid one. ${ }^{4}$ They are biodegradable, non-toxic and show good stability against coalescence, drug leakage, hydrolysis and particle growth. Also, they show high dispersibility in aqueous medium and may be loaded with hydrophobic drugs. Due to their solid matrix, they allow drug release over prolonged periods compared to nanoemulsions. ${ }^{5}$ Moreover, SLNs are made of physiological lipids and do not require organic solvents to be synthesized unlike polymeric nanoparticles, showing reduced toxicity and enhanced biocompatibility. ${ }^{6}$ 
In this work, Gelucire 4/14 was selected as lipid component due to its interesting properties. Gelucire $4 / 14$ (lauroyl polyoxylglycerides) is a mixture of PEG (polyethylene glycol) esters, free PEG and a small glyceride fraction and it has been widely used in pharmaceutical formulations as an amphiphilic component for improving water solubility of hydrophobic drugs and controlled release systems. It is considered as GRAS (generally regarded as safe) and its melting point is around $43-50{ }^{\circ} \mathrm{C}$.

Development of SLNs involves low cost and easiness for scaling-up, and production methods are suitable for large scale production.

Numerous studies have reported the use of lipid nanoparticles for dermal application of cosmetic and pharmaceutical active substances, such as coenzyme Q10, ascorbyl palmitate, tocopherol (vitamin E) and retinol (vitamin A). ${ }^{7}$ Chemical stability of such sensitive actives may be enhanced when incorporated into these drug carrier systems. In particular, the stabilization effect of SLN on retinol has been studied and it was suggested that stabilization depends on the type of lipids and surfactants used. Moreover, SLNs have shown increased UV-blocking ability and may be considered as physical sunscreens. ${ }^{8}$ Other advantages of SLNs include occlusive properties, increase in skin hydration, modified release profile, increase of skin penetration associated with a targeting effect and avoidance of systemic uptake. ${ }^{9}$ The small size and relatively narrow distribution of loaded-SLNs enable site specific delivery of lipophilic active compounds such as vitamin $\mathrm{A}$, and the close contact between the small particles and the stratum corneum, the primary barrier to dermal drug absorption, may increase the active concentration in the skin.

Several methods have been developed for the preparation of SLNs and basic production methods involve high pressure homogenization techniques. These techniques are based on the same procedures used in the preparation of solid lipid nanoemulsions, where lipids are mixed at temperatures 5-10 ${ }^{\circ} \mathrm{C}$ above their melting point. ${ }^{10}$ In order to overcome these problems, cold homogenization was developed. In cold homogenization, the lipid melt containing the active compound is cooled down before being passed through the high pressure homogenizer. Compared to other preparation techniques such as micro-emulsion and ultrasonication, high pressure homogenization presents major advantages including easy scale up, avoidance of organic solvents and short production times. ${ }^{6,11-16}$

In the present work we report a procedure for the synthesis of a novel formulation of vitamin A-loaded SLNs (SLN-A) for dermal delivery. Extensive characterization studies were conducted, including techniques such as dynamic light scattering (DLS), differential scanning calorimetry (DSC), Fourier transform infrared spectroscopy (FTIR), X-ray powder diffraction (XRPD) and small angle $\mathrm{X}$-ray scattering (SAXS). Additionally, we studied the entrapment efficiency (EE) and the stability of SLN-A by UV-Vis spectroscopy.

\section{Experimental}

\section{Materials}

Vitamin A palmitate and Gelucire $44 / 14^{\circledR}(\mathrm{G})$ were provided by Gattefossé (Gennevilliers, France). Cetyl alcohol (CA) and Tween $80^{\circledR}$ were provided by SigmaAldrich and sodium lauryl sulfate was purchased from Biopack. All other reagents and solvents used were of analytical grade.

\section{Methods}

\section{Preparation of SLN-A}

The lipid phase ( $2 \%$ formulation) was melted at $55{ }^{\circ} \mathrm{C}$. Vitamin A palmitate was dispersed in the lipid phase to obtain a clear solution. The dispersion medium (monosodium and disodium phosphate buffer $\mathrm{pH} 6.4$ with $0.2 \%$ Tween $80^{\circledR}$ ) was also heated at the lipid melting point $\left(\mathrm{T}=55^{\circ} \mathrm{C}\right)$, using the following sample composition: Gelucire $44 / 14^{\oplus}, 1.125 \%$, Vitamin A palmitate, $0.500 \%$; cetyl alcohol, $0.375 \%$; Tween $80^{\circledR}, 0.200 \%$ and water, $97.8 \%$. The hot lipid phase was emulsified by high speed stirring using a rotor-stator homogenizer (Ultra-turrax T25, IKA, Staufen, Germany). The dispersion was then homogenized using a high pressure homogenizer (Avestin Emulsiflex C5, Ottawa, Canada). Three cycles at 1000 bar were performed. The nanodispersion was stored at $4{ }^{\circ} \mathrm{C}$ to obtain solid lipid nanoparticles by recrystallization. ${ }^{17}$

\section{Freeze drying of SLN-A suspension}

In order to obtain a dry nanopowder, SLN-A was mixed with a $5 \%$ sucrose solution, frozen at $-70{ }^{\circ} \mathrm{C}$ in an ultra low temperature freezer (U410, New Brunswick Scientific Co Inc, Edison, New Jersey, USA) and lyophilized in a freeze dryer (Lyolab BII LSL Secfroid, Aclens-Lausanne, Switzerland) for 48 hours. The obtained powder was stored in a vacuum desiccator at room temperature for further characterization.

\section{Determination of vitamin A}

The determination of vitamin A palmitate was performed according to a modification of the method described in the 
United States Pharmacopeia (USP 30 NF 25).${ }^{18}$ Briefly, the method consisted on the dilution of SLN-A in isopropyl alcohol and its measurement at $325 \mathrm{~nm}$, the absorption peak of vitamin A palmitate, using an UV-Vis spectrophotometer (Ultrospec 3100 pro, Amersham Bioscience, Amersham, UK). A blank was prepared using isopropyl alcohol and the concentration of vitamin A palmitate was calculated from previously prepared calibration curves.

\section{Vitamin A entrapment efficiency}

The entrapment efficiency (EE) was expressed as the percentage of vitamin A palmitate entrapped with respect to the total vitamin A palmitate added. The entrapped vitamin content was calculated indirectly by determination of the free vitamin in the dispersion medium. Vitamin A content was determined by UV-Vis spectroscopy.

\section{Particle size and size distribution of SLN-A}

Hydrodynamic diameter and polydispersity index (PDI) of SLN-A were determined by dynamic light scattering using a particle size/zeta potential analyzer (Nano-ZS, Malvern Instruments Ltd., Worcestershire, UK). Measurements were performed in aqueous medium at $25^{\circ} \mathrm{C}$ and at an angle of $90{ }^{\circ} \mathrm{C}$. Samples were analyzed in triplicate and particle size and PDI were obtained by calculating the average of 3 measurements for each sample.

\section{Surface potential of SLN-A}

The zeta potential of SLN-A was determined according to laser Doppler electrophoretic mobility theory using the Nano-ZS (Malvern Instruments Ltd., Worcestershire, UK) at $25^{\circ} \mathrm{C}$. Zeta potential values were calculated from measured electrophoretic mobility, using the HelmoltzSmoluchowski equation. ${ }^{19}$ Samples were analyzed in triplicate and zeta potential was obtained by calculating the average of 3 measurements for each sample.

\section{DSC measurements}

A differential scanning calorimeter (DSC-60, Shimadzu, Kyoto, Japan) was used to determine the melting point of individual lipids, the lipid mixture, the lipid mixture containing vitamin A palmitate and SLN-A. Approximately $2 \mathrm{mg}$ of sample were weighed in aluminum pans and heated from 20 to $65^{\circ} \mathrm{C}$ with a temperature ramp rate of $5^{\circ} \mathrm{C} \mathrm{min}^{-1}$.

\section{FTIR studies}

Infrared analysis of samples was carried out using a Fourier transform infrared spectrophotometer (IR-Prestige 21, Shimadzu, Kyoto, Japan). Pellets of $\mathrm{KBr}$ (3\%) were analyzed over the range of $400-4000 \mathrm{~cm}^{-1}$ at a resolution of $4 \mathrm{~cm}^{-1}$, with an average of 20 scans.

\section{XRPD studies}

$\mathrm{X}$-ray powder diffraction was performed using a diffractometer (Ultima IV, Rigaku, Texas, USA) with CuK $\alpha$ radiation in the $2 \theta$ range of $5-50^{\circ}$, with a step size of $0.02^{\circ}$ and counting time of $2 \mathrm{~s}$ per step.

\section{SAXS studies}

Small angle X-ray scattering measurements were performed using the Ultima IV diffractometer (Rigaku, USA) using $\mathrm{CuK} \alpha$ radiation and covering a $\mathrm{q}$ range of 0.08-4.00 $\AA^{-1}$. The raw data was analyzed using the three-region Guinier-Porod model. ${ }^{20}$

\section{TEM observations}

Transmission electron microscopy (TEM) was performed with an electron microscope (Jem 1010, Jeol, Tokyo, Japan) operating at $100 \mathrm{kV}$ and images were captured with a digital camera (C4742-95, Hamamatsu, Hamamatsu City, Japan). The sample powder was dispersed in isopropyl alcohol and sonicated for $10 \mathrm{~min}$. A $5 \mu \mathrm{L}$ drop of the solution was deposited onto a copper grid coated with a holey carbon film.

\section{Separation of SLN-A}

The SLN-A suspension was separated from the free vitamin by gel filtration chromatography. Sufficient amount of gel Sephadex G-25 (Sigma, bead size 20-50) was previously soaked in distilled water and packed in a disposable polypropylene column that had a height of $7 \mathrm{~cm}$ and a diameter of $2 \mathrm{~cm}$. The column was sealed at both ends with porous polyethylene discs and calibrated with a mixture of Blue Dextran and dinitrophenyl lysine solution. The first fractions collected (void volume) corresponded to SLN-A, while free vitamin A palmitate eluted in the later fractions (bed volume). ${ }^{21}$

\section{Evaluation of SLN-A stability}

In order to assess the stability of SLN-A over time, two samples were prepared: (i) $10 \mathrm{~mL}$ of SLN-A and (ii) a suspension of vitamin A palmitate $(0.5 \% \mathrm{~m} / \mathrm{v})$ in monosodium and disodium phosphate buffer $\mathrm{pH} 6.4$, with $4 \%$ sodium lauryl sulfate. Samples were stored at $25^{\circ} \mathrm{C}$ and vitamin A content was monitored by UV-Vis spectroscopy at 1, 3, 5, 7 and 10 days after preparation. Prior to UV determination, free vitamin A was separated from SLN-A according to the above mentioned procedure.

\section{Results and Discussion}

High pressure homogenization is a suitable method for the preparation of homogeneous nanoparticulate systems 
with simple laboratory equipment. We herein developed a novel cosmetic formulation of SLNs for the topical delivery of vitamin A where cetyl alcohol (CA) and Gelucire $44 / 14^{\circledR}$ (G) were used as carrier materials. Gelucire $44 / 14$ is a mixture of glycerol and PEG 1500 esters of long-chain fatty acids, furthermore, since Gelucire 44/14 can form solid dispersions with drugs, its incorporation in lipid nanocarriers might be helpful in increasing the drug loading of hydrophobic drugs, especially those with a poor affinity for solid lipids. Additionally, the bioavailability enhancing properties of this amphiphilic excipient coupled with the nanoscale of lipid nanocarriers would offer greater therapeutic advantages. In view of this and the few works in topic applications, we evaluated the feasibility of fabricating SLN using Gelucire 44/14 as a stabilizer.

\section{Encapsulation efficiency}

Composition of the lipid is a main factor in the design of SLNs for the delivery of hydrophobic actives. ${ }^{11,22-25}$ The studied SLN-A system showed a high entrapment efficiency (EE), as shown in Table 1, with a mean EE of
$90.7 \pm 1.4 \%$. The obtained results may be attributed to the high lipophilicity of vitamin A palmitate and its good compatibility with the G+CA mixture.

Table 1. Entrapment efficiency of SLN-A

\begin{tabular}{lc}
\hline Sample name & EE / \% \\
\hline 1 & $90.9 \pm 0.5$ \\
2 & $91.3 \pm 0.4$ \\
3 & $89.8 \pm 0.4$ \\
Mean & $90.7 \pm 1.4$ \\
\hline
\end{tabular}

\section{Physicochemical characterization}

Particle size, size distribution (usually indicated by polydispersity index, PDI) and zeta potential measurements are quality parameters that depend on the preparation method and composition of SLNs systems, and are crucial in the production and stability of SLNs. ${ }^{26}$ Samples had an average PDI of $0.459 \pm 0.042$, suggesting bi-modal size distributions, as shown in Figure 1. According to the intensity and volume-based size distributions, two peaks
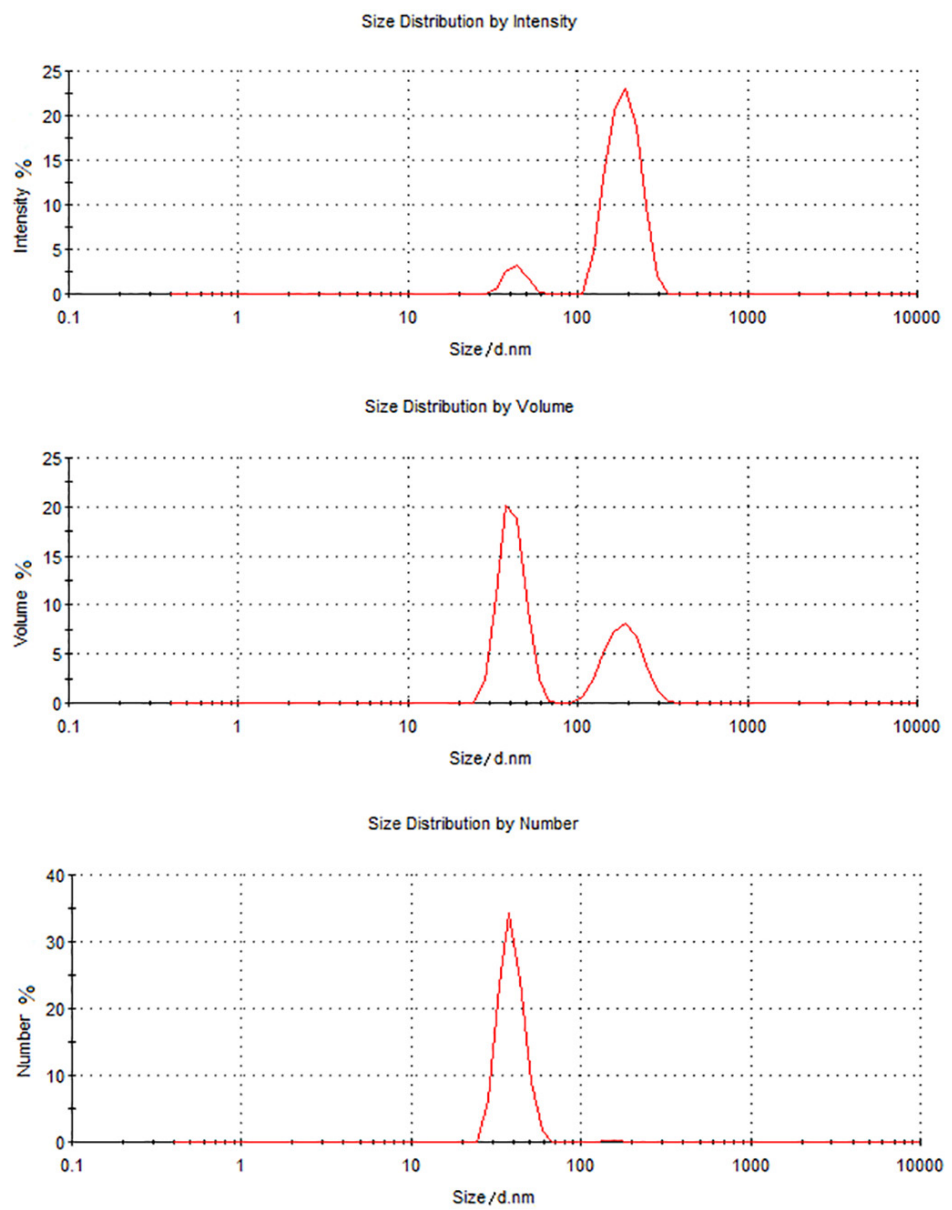

Figure 1. Particle size distributions by intensity, volume and number, measured at $25^{\circ} \mathrm{C}$ and $\mathrm{pH} 6.4$. 

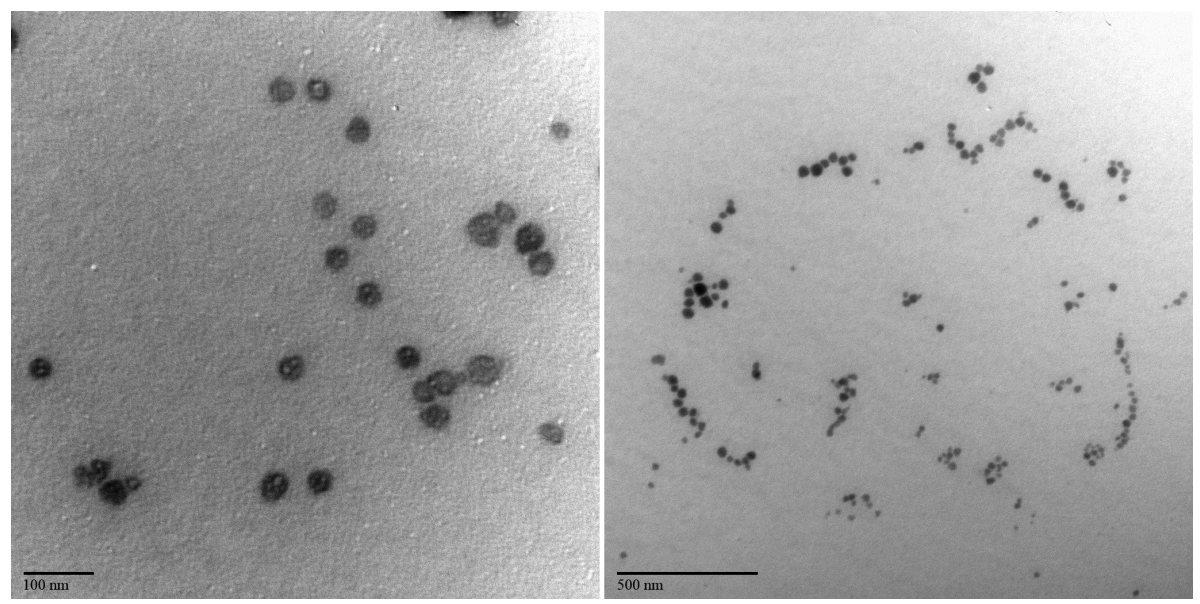

Figure 2. TEM images of SLN-A.

were observed at ca. 40 and $190 \mathrm{~nm}$. However, the numberbased distribution showed one peak, suggesting that the majority of the SLN-A particles $(99 \%)$ have a diameter size of ca. $40 \mathrm{~nm}$.

The magnitude of the zeta potential provides information about the surface charge of nanoparticles and can be used to predict colloidal stability of the studied system. Particles having a sufficiently high electrostatic repulsion resist flocculation and their colloidal system would be stable. Generally, particles with zeta potentials above $30 \mathrm{mV}$ (absolute value) are considered stable. In this case, the mean zeta potential value was of $-17.7 \pm 1.9 \mathrm{mV}$, indicating that the investigated SLN-A are slightly negatively charged with a relatively low zeta potential value. However, the studied nanoparticle suspensions were visually monitored over a period of 45 days and no phase separation was observed in this period. Despite having a lower absolute zeta potential, the system may tend to remain stable due to steric stabilization rather than electrostatic stabilization. ${ }^{27}$ In fact, the adsorption of nonionic stabilizers, such as Tween ${ }^{\circledR}$, onto SLNs surface may decrease the zeta potential as a result of the shift in the shear plane of the particle. ${ }^{28}$ For this reason, zeta potential cannot be considered a primary parameter to indicate the colloidal stability of SLN-A. ${ }^{29}$

Further indications for particle aggregation can be obtained from X-ray diffraction studies. ${ }^{30}$

As shown in the TEM observations displayed in Figure 2, the SLN-A sample exhibited good homogeneity and particles had a spherical morphology. Size measurements obtained from TEM images showed that particles had an average diameter size of about 30-50 nm, resulting in good agreement with the previous DLS findings.

Figure 3 shows the results of the SAXS analysis using the Guinier-Porod method. The Guinier fit for spherical particles showed the presence of a population of scatterers with diameter ca. $55 \mathrm{~nm}$ in the low-Q region. In the

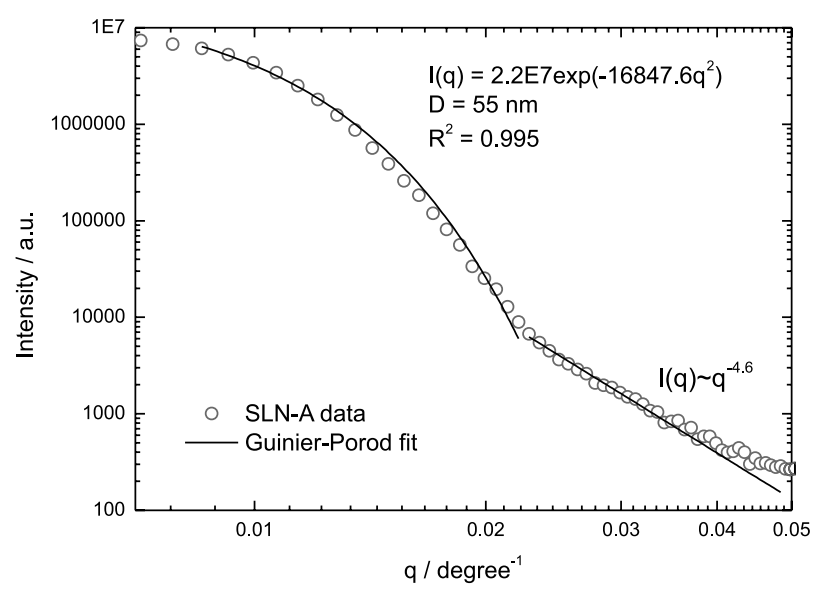

Figure 3. SAXS curve and Guinier-Porod fit of SLN-A.

intermediate- $Q$ region, a power-law regime was observed with an $\mathrm{I}(\mathrm{q}) \sim \mathrm{q}^{-4.6}$ dependence showing a slight negative deviation from Porod's law, in good agreement with a non-ideal quasi two-phase system.

$\mathrm{X}$-ray diffraction patterns for $\mathrm{G}, \mathrm{CA}, \mathrm{G}+\mathrm{CA}$ and SLN-A samples are shown in Figure 4. It can be seen that both $\mathrm{G}+\mathrm{CA}$ and SLN-A patterns are not the simple sum of $\mathrm{G}$ and CA.

The hkl peak positions of all samples are presented in Table 2. G hkl reflections in G+CA and SLN-A patterns showed very low $2 \theta$ shifts compared to pure $G$ hkl reflections, which have been previously reported at $2 \theta$ ca. 19.1 degrees. ${ }^{31}$ On the other hand, CA reflections observed in the G+CA and SLN-A patterns showed a relevant shift from $2 \theta=21.70$ degrees towards lower values at $2 \theta=21.28$ degrees. This observation denotes a significant compression in the CA unit cell parameters when blended with $\mathrm{G}$, suggesting that the two phases are well mixed.

Crystallite sizes determined using Scherrer equation is shown in Table 3. A significant decrease in the crystallite diameter was observed for both the G+CA mixture and the 


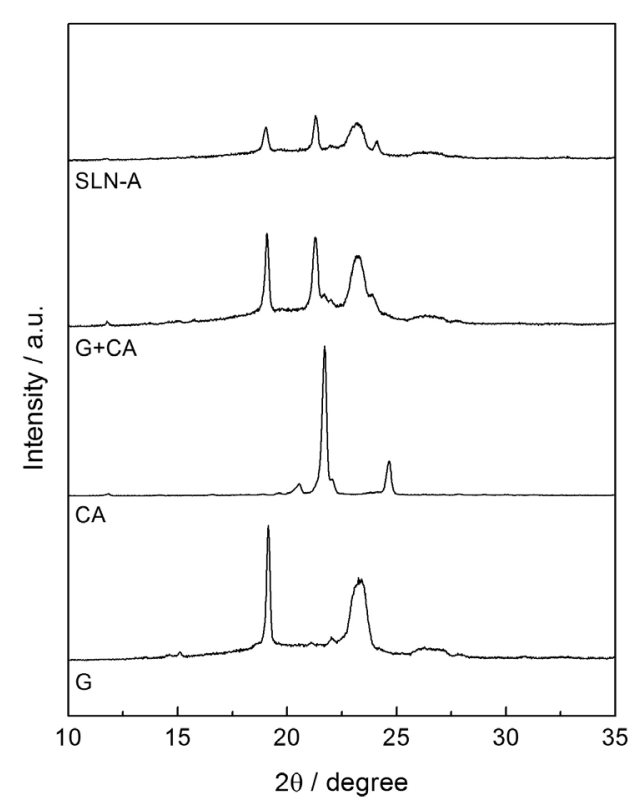

Figure 4. XRPD pattern for Gelucire (G), cethyl alcohol (CA), G+CA and SLN-A nanoparticles.

Table 2. Crystallite diameter (D) calculated using Scherrer equation of $\mathrm{CA}, \mathrm{G}, \mathrm{G}+\mathrm{CA}$ and SLN-A

\begin{tabular}{|c|c|c|c|c|}
\hline & $\mathrm{CA}$ & G & $\mathrm{G}+\mathrm{CA}$ & SLN-A \\
\hline \multicolumn{5}{|l|}{ CA hkl } \\
\hline $2 \theta$ position / degree & 21.70 & - & 21.28 & 21.28 \\
\hline $\mathrm{d}$ spacing / $\mathrm{A}$ & 4.09 & - & 4.17 & 4.17 \\
\hline Diameter / nm & $>200$ & - & 85.2 & 90.3 \\
\hline \multicolumn{5}{|l|}{ G hkl } \\
\hline $2 \theta$ position / degree & - & 19.14 & 19.08 & 19.02 \\
\hline d spacing / $\AA$ & - & 4.64 & 4.65 & 4.66 \\
\hline Diameter / nm & - & 50.9 & 39.8 & 33.3 \\
\hline
\end{tabular}

vitamin A-loaded nanoparticles (SLN-A) compared to the isolated components.

According to Figure 5, the obtained FTIR spectra for sample $\mathrm{G}$ showed two intense characteristic peaks at 1736 and $1117 \mathrm{~cm}^{-1}$, attributed to the $\mathrm{C}=\mathrm{O}$ stretching mode of ester groups and the $\mathrm{C}-\mathrm{O}$ stretching mode of alcohol groups, respectively.

Other minor intensity peaks were also observed, in good agreement with the literature. ${ }^{32} \mathrm{CA}$ spectra showed three characteristic peaks at 1464, 1063 and $719 \mathrm{~cm}^{-1}$, attributed to the $\mathrm{CH}_{2}$ bending of the aliphatic chain, and the $\mathrm{C}-\mathrm{O}$ and $\mathrm{O}-\mathrm{H}$ stretching of the alcohol group, respectively.

There was no significant difference between the melting point of G+CA and SLN-A, suggesting no partial decomposition in the preparation process. Furthermore, no clear evidence of vitamin A was observed in the FTIR spectra of SLN-A, probably due to its low concentration

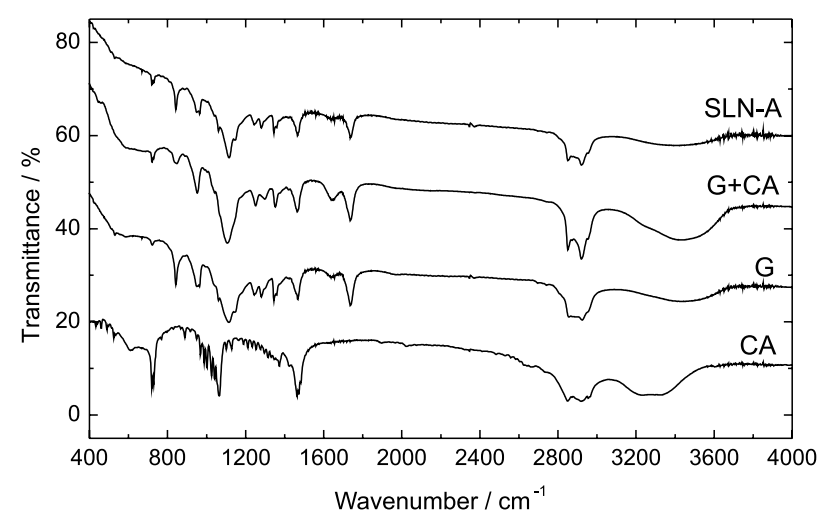

Figure 5. FTIR spectra of Gelucire (G), cetyl alcohol (CA), G+CA and SLN-A.

that may go undetected by this characterization technique.

DSC was used to detect interactions between $\mathrm{G}$ and $\mathrm{CA}$. DSC curves showed the presence of endothermic peaks that corresponded to the melting points of the individual and the blended components of the formulation, indicating an interesting effect of $\mathrm{G}$ content on the thermal transition of CA. In the heating cycle, the endothermic transition corresponding to the melting point of $\mathrm{CA}$ ranged between 39 and $44{ }^{\circ} \mathrm{C}$. There are potential interferences between CA and $\mathrm{G}$ in the melting process of $\mathrm{CA}$ that affect the physical construction of the resulting SLN-A.

The SLN-A and G+CA melting points shifted towards lower temperatures with respect to the individual components. As it is observed in Figure 6, the melting points are ca. $51^{\circ} \mathrm{C}$ for $\mathrm{CA}$, ca. $45^{\circ} \mathrm{C}$ for $\mathrm{G}$ and ca. $40{ }^{\circ} \mathrm{C}$ for $\mathrm{G}+\mathrm{CA}$ and SLN-A.

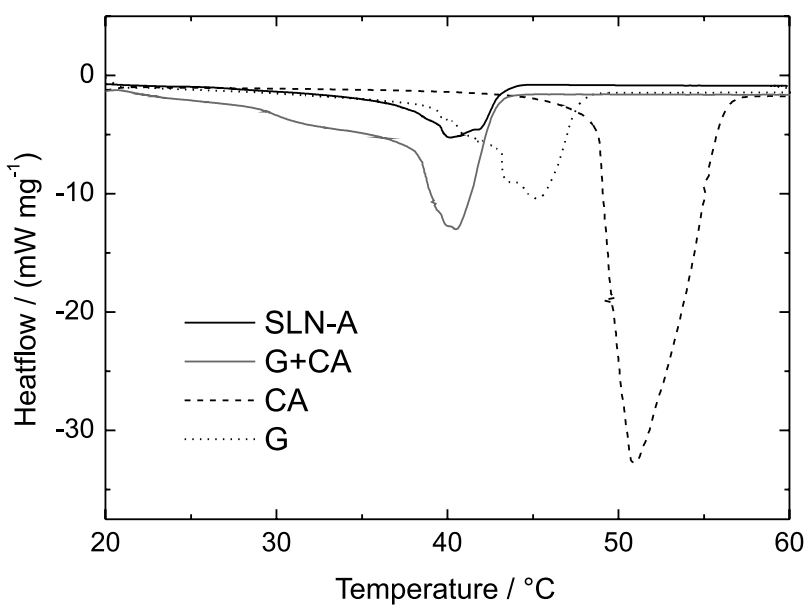

Figure 6. DSC curves of Gelucire (G), cetyl alcohol (CA), G+CA and SLN-A.

This is probably due to the decrease in crystallinity and the lower fusion enthalpy of the G+CA mixture caused by the combination of the components. Additionally, SLN-A showed a higher decrease in fusion enthalpy compared to 
the G+CA mixture, suggesting a lower crystallinity that is typically observed in nanoparticulated systems. These observations are also in good agreement with the previously discussed results from XRPD analysis. Both the decreasing size and the increasing degree of imperfections in the crystal play an important role in charge capacity.

To investigate the protection conferred by the studied SLN system to the encapsulated active, we monitored vitamin A content during 10 days as an indicator of vitamin chemical stability. As shown in Figure 7, the lipid carrier system conferred high protection to vitamin A, resulting in about $70 \%$ loss during the first 72 hours, compared to about $20 \%$ degradation when the unprotected vitamin was studied at pH 6.4.

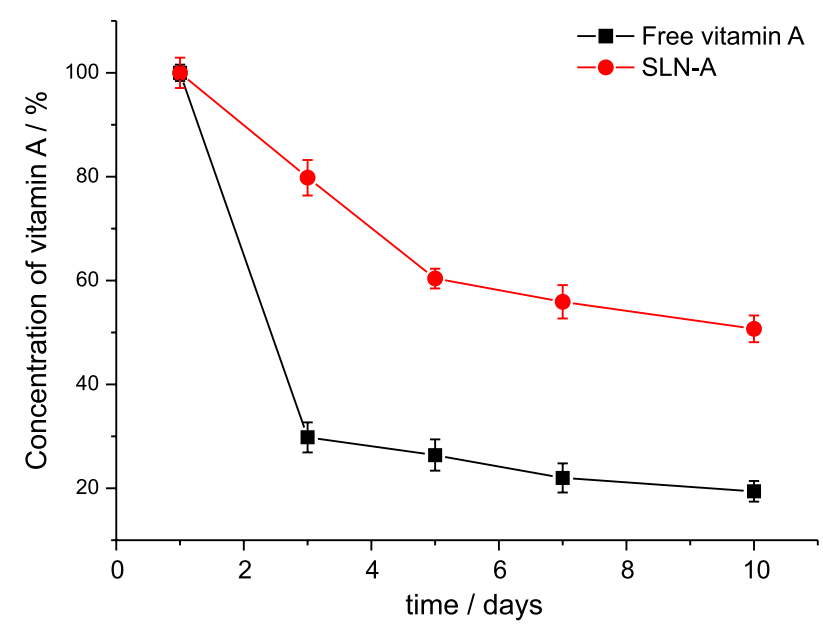

Figure 7. Vitamin A stability test at $25^{\circ} \mathrm{C}$ and $\mathrm{pH}$ 6.4: SLN-A and free vitamin A. Solid lines are a guide to the eye.

Moreover, SLNs were able to protect the vitamin A for a longer period of time, reaching a degradation of about $50 \%$ only after 10 days.

Hence, these results suggest that the investigated SLN formulation may be an effective way to retard vitamin A degradation.

\section{Conclusions}

A novel formulation of vitamin A-loaded SLNs, containing Gelucire $44 / 14^{\circledR}$ and cetyl alcohol, was successfully prepared by the hot homogenization method. Various techniques were used to evaluate the composition, morphology and crystallinity of the nanoparticles, as well as other physicochemical characteristics. The studied system showed high encapsulation efficiency and conferred protection against degradation of vitamin A. Furthermore, the lipids in these SLNs showed a melting temperature very close to normal body temperature, which makes them suitable for later application in topical application of vitamin A.

\section{Acknowledgments}

The authors would like to thank PEDECIBA, ANII, CSIC, INNOVA-EU and CINQUIFIMA for financial support.

\section{References}

1. Pallavi, P. V.; Kamalinder, S. K.; AAPS Pharm. Sci. Tech. 2006, 7, 91 .

2. Carlotti, M. E.; Rossatto, V.; Gallarate, M.; Trotta, M.; Debernardi, F.; J. Cosmet. Sci. 2004, 55, 233.

3. Müller, R. H.; Radtke, M.; Wissing, S. A.; Bull. Tech. Gattefosse 2002, 131.

4. Ekambaram, P.; Sathali, A.; Priyanka, K.; Sci. Rev. Chem. Commun. 2012, 2, 80.

5. Utreja, S.; Jain, N. K.; Advances in Controlled and Novel Drug Delivery, $1^{\text {st }}$ ed.; CBS Publishers: New Delhi, India, 2001.

6. Battaglia, L.; Gallarate, M.; Panciani, P. P.; Ugazio, E.; Sapino, S.; Peira, E.; Chirio, D.; Application of Nanotechnology in Drug Delivery; Sezer, A. D., ed.; InTech Open Access, 2014.

7. Guimarães, K.; Ré, M.; Nanocosmetics and Nanomedicines, $1^{\text {st }}$ ed.; Beck, R.; Guterres, S.; Pohlmann, A., eds.; Springer: Berlin, Germany, 2011.

8. Wissing, S. A.; Müller, R. H.; Int. J. Pharm. 2003, 18, 254.

9. Preeti, K. S.; Prameet, S.; Shailendra, S.; Afr. J. Pharm. Pharmacol. 2013, 7, 138.

10. Pardeike, J.; Hommoss, A.; Müller, R. H.; Int. J. Pharm. 2009, 366, 170.

11. Müller, R. H.; Mäder, K.; Gohla, S.; Eur. J. Pharm. Biopharm. 2000, 50, 161.

12. Jenning, V.; Lippacher, A.; Gohla, S.; J. Microencapsulation 2002, 19, 1.

13. Battaglia, L.; Gallarate, M.; Expert Opin. Drug Delivery 2012 , 9, 497.

14. Müller, R. H. In Handbook of Pharmaceutical Controlled Release Technology; Wise, D., ed.; Marcel Dekker Inc.: New York, USA, 2000, ch. 18.

15. Jahnke, S. In Emulsions and Nanosuspensions for the Formulation of Poorly Soluble Drugs; Müller, R. H.; Benita, S.; Böhm, B., eds.; Medpharm Scientific Publishers: Stuttgart, Germany, 1998, ch. 10.

16. Mehnert, W.; Mäder, K.; Adv. Drug Delivery Rev. 2001, 47, 165.

17. Shi, F.; Zhao, J. H.; Liu, Y.; Wang, Z.; Zhang, Y. T.; Feng, N. P.; Int. J. Nanomed. 2012, 7, 2033.

18. The United States Pharmacopeia, USP 30-NF 35; The United States Pharmacopeial Convention: Rockville, 2007.

19. Poyton, M. F.; Cremer, P. S.; Anal. Chem. 2013, 85, 10803.

20. Hammouda, B.; J. Appl. Crystallogr. 2010, 43, 716.

21. Ackers, G. K.; Biochemistry 1964, 3, 723. 
22. Müller, R. H.; Dingler, A.; Eurocosmetic 1998, 7-8, 26.

23. Hajare, A.; Mali, S.; Ahir, A.; Thorat, J.; Salunkhe, S.; Nadaf, S.; Bhatia, N.; J. Adv. Drug Delivery 2014, 1, 30.

24. Tzachev, C.; Svilenov, H.; Int. J. Pharm. Sci. Rev. Res. 2013, $18,103$.

25. Shah, R.; Lipid Nanoparticles: Production, Characterization and Stability; Springer International Publishing, 2015.

26. Shah, R.; Eldridge, D.; Palombo, E.; Harding, I.; J. Phys. Sci. 2014, 25, 59.

27. Kashanian, S.; Azandaryani, A. H.; Derakhshandeh, K.; Int. J. Nanomed. 2011, 6, 401.
28. Jenning, V.; Schäfer-Korting, M.; Gohla, S.; J. Controlled Release 2000, 66, 115.

29. Shimpi, S. L.; Chauhan, B.; Mahadik, K. R.; Paradkar, A.; Pharm. Res. 2005, 22, 1727.

30. Damian, F.; Blaton, N.; Naesens, L.; Balzarini, J.; Kinget, R.; Augustijns, P.; Eur. J. Pharm. Sci. 2000, 10, 311.

31. Parmar, K. R.; Shah, S. R.; Sheth, N. R.; Dissolution Technol. 2011, 34, 55.

32. Karataş, A.; Yüksel, N.; Baykara, T.; Farmaco 2005, 60, 777.

Submitted: May 20, 2016

Published online: October 14, 2016 